\title{
STEREOLOGICAL ESTIMATES FOR ROUGHNESS AND TORTUOSITY IN CEMENTITIOUS COMPOSITES
}

\author{
PIET STROEVEN \\ Faculty of Civil Engineering and Geosciences, Stevinweg 1, 2600 GA, Delft, The Netherlands \\ (Accepted January 28, 2000)
}

\begin{abstract}
Relatively weak interfaces between aggregate grains and the cementitious matrix initiate the damage evolution process leading to fracture. Coalescence between nearby interface cracks is promoted by the small nearest neighbour distances in a dense random packing of the aggregate. The fracture surface is therefore modelled as a dividing plane from which particles protrude. Assuming spherical aggregate, roughness is obtained as the global geometrical-statistical expression for the increase in fracture surface area due to a multitude of dome-like caps of various sizes. Transport phenomena in concrete are equally influenced by the aggregate, because traversing water-born molecules or ions have to go around the dense grains. This route is additionally promoted by the relatively high porosity in the interfacial transition zone. The planar and linear concepts of tortuosity in the transport path are analogous to those of roughness.
\end{abstract}

Keywords: cementitious materials, fracture surface, particle-matrix interface, particle size distribution, roughness, tortuosity.

\section{INTRODUCTION}

Cementitious materials belong to a class of macroscopically heterogeneous materials. They can be conceived at the meso-level of the microstructure as densely packed multi-sized relatively dense, stiff and strong particles of an aggregate stabilized by a cementitious binder. This particulate component constitutes an effective load-bearing skeleton in the compression domain. The wider the range of particle sizes employed in the aggregate or in the (blended) cement, the higher the total packing density which can be achieved (Jiang and Roy, 1994). Instead, under tensile loads, the skeleton will be separated into portions by yielding of the bond between groups of particles and the cementitious binder, the latter being the weakest link in the micro-mechanical system. A single fracture surface is resulting, which bears evidence of the particulate nature of the material. An otherwise flat or slightly curved plane, the so called dividing plane, reveals protruding particles that were in the path of the plane/crack. The morphological characteristics (texture) of the fully developed fracture surface can therefore be considered a 'fingerprint' of the material. Under compressive loads, generally, several fracture surfaces are formed, but the material's identity will be similarly reflected by the textural features of these fracture surfaces. Roughness is frequently employed to define surface texture in a global way.
Research has demonstrated the concept of a particlematrix interface to be more complex in cementitious materials. In reality, a very thin interphase layer is formed around particles (of, say, $50 \mu \mathrm{m}$ ). This layer is commonly attributed as Interfacial Transition Zone (ITZ). The existence of the ITZ can be considered leading to a second order effect on the estimation of roughness of a fracture surface. This means that the fracture path may not always follow the supposedly smooth surface of the aggregate particle, but may deviate into the ITZ. The relatively high porosity in the ITZ has relevance for durability, since it governs the rate of ingress of gaseous or fluid substances into the concrete. For the estimation of tortuosity of the transfer route, the existence of the ITZ can be considered yielding a second order influence, as well (Jaiswal et al., 1997). The simplest morphological concept reflecting the transport and fracture paths consists of a flat plane that is only curved around particle obstacles. The analytical approach in this paper is based on this physical model.

This surface of macroscopical dimensions, shown in Fig. 1, is composed of inter-connected planar parts of a dividing plane, and of dome-like caps and indentations of spherical particles intersecting with the plane. Because of obvious reasons, the largest part of each particle is embedded. In transport or fracture problems alike, the textural 'finger print' originating from the large number of caps and indentations should be quantified. 


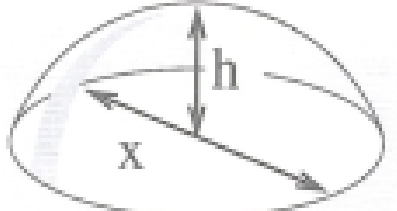

dividing plane

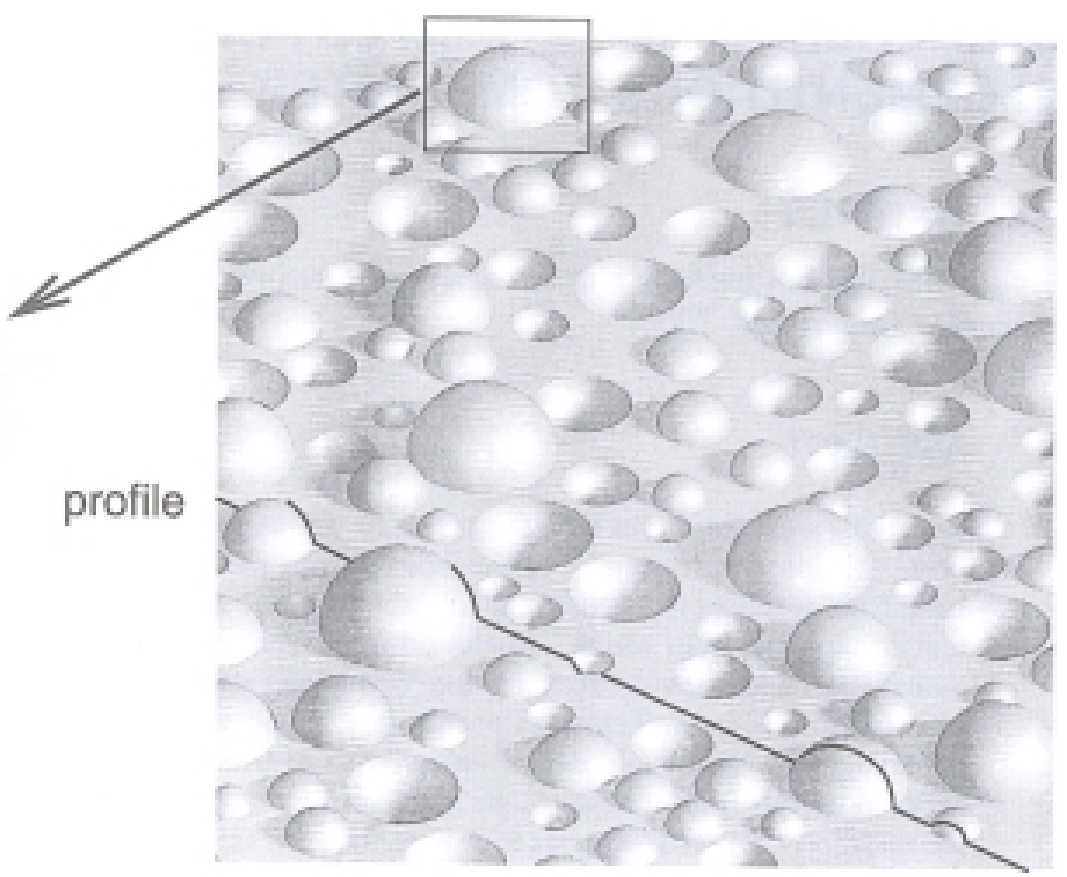

Fig. 1. Fracture surface or transport path in a computer-simulated concrete; a fracture or transport profile is additionally indicated (see Stroeven, 1999).

\section{TEXTURE ANALYSIS}

The size of the intersection circle and the height of the cap of a particle intersecting with the dividing surface are denoted by $x$ and $h$, respectively, as shown in Fig. 1. The solution to the morphological problem is governed by the density functions of $x$ and $h$, defined by $\phi(x)$ and $\psi(h)$, respectively. Since all values of $h$ between 0 and $d / 2$ are equally likely, we have $\psi(h)=2 / d$, in which $d$ is particle size. Before generalizing the approach, a mono-sized aggregate is considered. Hence, $d=$ constant. The first and second moment of $\psi(h)$ are, respectively

$\bar{h}=\frac{2}{d} \int_{0}^{\frac{d}{2}} h \mathrm{~d} h=\frac{d}{4}$ and $\overline{h^{2}}=\frac{2}{d} \int_{0}^{\frac{d}{2}} h^{2} \mathrm{~d} h=\frac{d^{2}}{12}$

$\phi(x)$ is governed by an integral equation of Abel's type (Stroeven, 1973; Kendall and Moran, 1963)

$$
\phi(x)=\frac{x}{\bar{d}} \int_{x}^{d_{m}} \frac{f(d)}{\sqrt{d^{2}-x^{2}}} \mathrm{~d} d
$$

in which $d_{m}$ can be associated with the largest particles in the aggregate. $f(d)$ is the particle size distribution function (pdf). For mono-sized spheres, eq (2) reduces to (Stroeven, 1973, 1982).

$$
\phi(x)=\frac{x}{d \sqrt{d^{2}-x^{2}}}
$$

The literature (Kendall and Moran, 1963) offers a direct relationship between the moment generation functions of $x$ and $d$, however, which simplifies the calculation of the moments of $\phi(x)$. The derivation is presented in Stroeven (1973).

$$
m_{n}(x)=M_{n}(d) \int_{0}^{\frac{\pi}{2}}(\sin \phi)^{n+1} \mathrm{~d} \phi
$$

in which $n$ indicates the number of the moment and $m_{n}(x)$ and $M_{n}(d)$ denote the moment generating functions of $x$ and $d$, respectively. For mono-sized spheres it is found that

$$
\begin{aligned}
\bar{x} & =m_{1}(x)=\frac{\pi}{4} M_{1}(d)=\frac{\pi}{4} d \\
\overline{x^{2}} & =m_{2}(x)=\frac{2}{3} M_{2}(d)=\frac{2}{3} d^{2}
\end{aligned}
$$

The surface area of a dome-like cap of a sphere is $S=\pi\left(h^{2}+\frac{1}{4} x^{2}\right)$ (Stroeven, 1982). The average surface area of all caps amounts

$$
\bar{S}=\pi\left(\overline{h^{2}}+\frac{1}{4} \overline{x^{2}}\right)=\frac{\pi}{4} d^{2}
$$

With $N$ as the number of caps, and $A$ as the area of the dividing plane, the density of caps in this plane is $N_{A}=6 V_{V} / \pi d^{2}$. The total surface area per unit of the dividing plane, $S_{A}$ is therefore

$$
S_{A}=\bar{S} \cdot N_{A}=\frac{3}{2} V_{V}
$$

$V_{V}$ in the last two relationship denotes the volume fraction of the aggregate.

Eq (7) holds for all size fractions in an arbitrary 
mixture of grains. Hence, averaging over all size fractions will yield the same outcome. This result can be obtained straight forward by a generalized approach to an arbitrary aggregate mixture. Let $f(d)$ define the particle size distribution function. The equivalent of the left hand side of eq (7) for a multi-sized mixture is

$$
\bar{S}_{A}=\overline{\bar{S}}_{A}=\int_{d_{o}}^{d_{m}} \bar{S}\left(d_{i}\right) N_{A_{i}}\left(d_{i}\right) \mathrm{d} d_{i}
$$

in which $N_{A}\left(d_{i}\right)=d_{i} N_{\lambda}\left(d_{i}\right)$ for a fraction of particles with average size $d_{i}$ and a very narrow size range.

With eq (6), this yields

$$
\bar{S}\left(d_{i}\right) N_{A}\left(d_{i}\right)=\frac{\pi}{4} d_{\mathrm{i}}^{3} N_{V}\left(d_{i}\right)
$$

and upon substitution in eq ( 8 )

$$
\bar{S}_{A}=\int_{d_{o}}^{d_{m}} \frac{\pi}{4} d_{i}^{3} N_{V}\left(d_{i}\right) f\left(d_{i}\right) \mathrm{d} d_{i}=\frac{3}{2} V_{V}
$$

\section{EXAMPLES FOR CONCRETE MIXTURES}

It has been shown earlier (Stroeven, 1983), that for continuously-graded aggregate mixtures for concrete, the particle size distribution function (psd) can generally be formulated by

$$
f(d)=m \frac{d_{o}^{m}}{d^{m+1}}
$$

where $d_{o}$ defines as before the smallest particles in the aggregate observed in an experimental approach, or included in a model. $d_{o}$ therefore defines the sensitivity of the concept. For $m=2.5$ or $m=3$ in eq (11), a so called Fuller mix (FU) or an equal volume fractions mix (EV) are obtained, which constitute mixes near the boundaries of the area for accepted mixes in the building codes.

Table 1 lists the first, second and third moments of the moment generation function

$$
M_{n}(d)=\overline{d^{n}}=\int_{d_{o}}^{d_{m}} d^{n} f(d) \mathrm{d} d
$$

for the FU and EV mixes (i.e. $f(d)$ according to eq (11) for $m=2.5$ and $m=3$, respectively). Further, the first and second moment of he cap height, $h$, and span, $x$, are presented. Finally, the expressions for $\bar{S}$ and $N_{A}$ are given. Use has been made of eq (6) and $N_{A}=6 \bar{d} V_{V} / \pi \overline{d^{3}}$

Table 1 shows that of the two, the equal volume fractions mix (EV) contains the largest amount of fines.

By multiplication of the expressions listed in the last two columns of Table 1, the expression in eqs (7) and (10) is regained. Hence, $S_{A}$ is indeed not depending on the details of the actual sieve curve.

\section{ROUGHNESS AND TORTUOSITY}

The planar index of surface roughness or tortuosity, $R_{s}$, is defined as the ratio of total surface area and the corresponding (projected) area of the dividing plane. Hence, planar values of roughness and of tortuosity are given by

$$
R_{S}=A_{A m}+S_{A}=1+S_{A}-V_{V}=1+\frac{1}{2} V_{V}
$$

in which $A_{A m}$ represents the areal fraction of the matrix along the divided plane. The linear index of roughness is analogously defined. A so called vertical section, perpendicular to the dividing plane, delineates a profile in the surface, as shown in Figure 1, at the right. The linear roughness index equals the ratio of the length of the profile and the corresponding (projected) length in the divided plane. The present model concept of a multisized spherical aggregate in a cementitious matrix allows also to derive an expression for the linear index of roughness along the profile. The fraction of the profile along the dividing plane is given by $L_{L m}=(1-$ $L_{L}$ ), where $L_{L m}=A_{A m}$. The fraction of the curved profile is proportional to $L_{L}$, and to the ratio of average values of circular perimeter length and its span. Hence, the linear roughness index $R_{l}$ for a mono- as well as a multisized aggregate can be expressed by

$$
R_{l}=1-L_{L}+\frac{\bar{\phi}}{\overline{\sin \phi}} L_{L}
$$

where $L_{L}=A_{A}=V_{V}$, and

$$
\bar{\phi}=\frac{\int_{0}^{\frac{\pi}{2}} \phi \mathrm{d} \phi}{\int_{0}^{\frac{\pi}{2}} \mathrm{~d} \phi}=\frac{\pi}{4} \text { and } \overline{\sin \phi}=\frac{\int_{0}^{\frac{\pi}{2}} \sin \phi \mathrm{d} \phi}{\int_{0}^{\frac{\pi}{2}} \mathrm{~d} \phi}=\frac{2}{\pi}
$$

Substitution in eq (13) yields

$$
R_{l}=1-A_{A}+\frac{\pi^{2}}{8} A_{A}=1+\left(\frac{\pi^{2}}{8}-1\right) A_{A}
$$

According to Underwood (1987), the estimate for $R_{s}$ would be

$$
R_{s} \approx \frac{4}{\pi}\left(R_{l}-1\right)+1
$$

This leads to $R_{s} \approx 1+0.298 V_{V}$. Coster and Chermant (1987) have proposed the relationship

$$
R_{s} \approx \frac{7}{4} R_{l}-\frac{3}{4}
$$

resulting in $R_{s} \approx 1+0.409 V_{V}$.

It can be observed, that for the present case the last estimate is closer to the exact expression given by eq (12). 
Table 1. Moments of the psd's according to eq (11) for $m=2.5$ (FU) and $m=3$ (EV), and of cap height, $h$ and span, $x$, are successively listed. Further, average cap surface area, $\bar{S}$, and number of caps per unit area of the dividing plane, $N_{A}$, are given. $d_{o}$ and $d_{m}$ are the smallest, respectively, the largest particles in the model.

\begin{tabular}{llllllllll}
\hline psd & $\bar{d}$ & $\overline{d^{2}}$ & $\overline{d^{3}}$ & $\bar{x}$ & $\overline{x^{2}}$ & $\bar{h}$ & $\overline{h^{2}}$ & $\bar{S}$ & $\mathrm{~N}_{\mathrm{A}}$ \\
\hline $\mathrm{FU}$ & $\frac{5}{3} d_{o}$ & $5 d_{o}^{2}$ & $5 d_{o}^{2.5} d_{m}^{0.5}$ & $\frac{5 \pi}{12} d_{o}$ & $\frac{10}{3} d_{o}^{2}$ & $\frac{5}{12} d_{o}$ & $\frac{5}{12} d_{o}^{2}$ & $\frac{3 \pi}{4} d_{o}^{1.5} d_{m}^{0.5}$ & $\frac{2}{\pi} V_{V} d_{o}^{-1.5} d_{m}^{0.5}$ \\
$\mathrm{EV}$ & $\frac{3}{2} d_{o}$ & $3 d_{o}^{2}$ & $3 d_{o}^{3} \ln \frac{d_{m}}{d_{o}}$ & $\frac{3 \pi}{8} d_{o}$ & $2 d_{o}^{2}$ & $\frac{3}{8} d_{o}$ & $\frac{1}{4} d_{o}^{2}$ & $\frac{\pi}{2} d_{o}^{2} \ln \frac{d_{m}}{d_{o}}$ & $\frac{3}{\pi} V_{V}\left(d_{o}^{2} \ln \frac{d_{m}}{d_{o}}\right)^{-1}$ \\
\hline
\end{tabular}

\section{CONCLUSIONS}

Stereological notions, based on geometrical probability theory, render possible the unbiased estimation of linear and planar measures for fracture surface roughness and for tortuosity in transport routes in cementitious materials. The solutions that are obtained in this way are presented in eqs (12) and (15). They are based on a common modelling concept in concrete technology, i.e. of a dense random packing of spherical aggregate particles and a (flat) dividing plane. The particle-matrix interface is adopted as the weakest link in the micro-mechanical system, in accordance with experimental evidences for conventional concrete qualities. The exact estimates for the linear and planar concepts allow an evaluation of approximate estimates for the planar rougness or tortuosity based on profile measurements. A formula proposed by Coster and Chermant yielded for the present case the best results.

The analytical solution to the planar case can be applied in modelling concepts underlying fracture or durability studies. Measurements can more easily be performed on profiles, however. So, the linear solution for roughness and tortuosity can be used in experimental approaches. The presented expressions in this paper allow, moreover, to transfer in a straight forward manner the experimental observations into exact information on the planar concept. This can be seen by subtracting eqs (12) and (15) yielding

$$
R_{s}=R_{l}+\left(\frac{3}{2}-\frac{\pi^{2}}{8}\right) V_{V} .
$$

This paper proves that roughness and tortuosity depend on the total amount of aggregate in the cementitious material, but not on the details of the sieve curve. This is of paramount importance in concrete technology in the fields of fracture mechanics as well as of concrete durability for setting up experiments and for correctly interpreting the obtained data.

A preliminary report of some of the data (Stroeven, 1999) has been presented at the $X^{\text {th }}$ International Congress for Stereology, Melbourne, Australia, 1-4 November 1999.

\section{REFERENCES}

Coster M, Chermant J-L (1983). Recent developments in quantitative fractography. Metals Reviews 28:228-50.

Jaiswal SS, Igusa T, Styer T, Karr A, Shah SP (1997). Influence of microstructure and fracture on the transport properties in cement-based materials. In: Brandt AM, Li VJ, Marshall IH, eds. Brittle Matrix Composites 5. Cambridge: Woodhead Publ. Ltd, 199-220.

Jiang W, Roy DM (1994). Strengthening mechanisms of high-performance concrete. In: Malhotra VM, ed. High Performance Concrete. Detroit: Proc ACI Int Conf Singapore, ACI, 753-67.

Kendall MG, Moran PAP (1963). Geometrical Probability. London: Griffin \& Co.

Stroeven P (1973). Some aspects of the micro-mechanics of concrete, PhD Thesis, Delft University of Technology, Delft.

Stroeven P (1982). Structural modelling of plain and fibrereinforced concrete; a morphological approach to a cracked region. Composites 13:129-39.

Stroeven P (1999). Study of crack development as the basis for rheology of cementitious materials. In: Wang R, ed. Proc IUTAM Symp Rheology of Bodies with Defects. Dordrecht: Kluwer Ac Publ, 205-22.

Underwood EE (1987). Stereological analysis of fracture roughness parameters. Acta Stereol 6(suppl II):170-8. 